\title{
Los distintos rostros de la inclusión y la exclusión
}

\author{
Aldo Mascareño y Fabiola Carvajal
}

RESUMEN

Los conceptos de inclusión y exclusión tienen una larga tradición en sociología y una importancia más bien reciente aunque significativa para el análisis de políticas públicas. Sin embargo, una cierta falta de flexibilidad conceptual aparece al aplicar la distinción a situaciones sociales complejas. En el artículo se analizan los principales enfoques de inclusión/exclusión en la tradición sociológica, la teoría sistémica y la teoría de las nuevas desigualdades. Sobre esta base, se construyen cinco situaciones interrelacionadas de inclusión y exclusión (autoinclusión/autoexclusión, inclusión por riesgo/exclusión por peligro, inclusión compensatoria, inclusión en la exclusión y subinclusión), al tiempo que se las ilustra con ejemplos específicos para desarrollar un refinamiento analítico de los problemas de inclusión y exclusión que pueda aportar al diagnóstico sociológico y a la evaluación de las consecuencias de decisiones públicas y privadas. Chile. aldo.mascareno@uai.cl

Fabiola Carvajal es asistente de investigación de la Escuela de Gobierno de la Universidad Adolfo Ibáñez, Santiago, Chile. fabi.casep@hotmail.com 


\section{I}

\section{Introducción}

En el análisis y práctica de políticas públicas, el uso de los conceptos de inclusión y exclusión comenzó a adquirir popularidad en los años noventa del siglo XX, especialmente en el contexto europeo y en el marco de acción de organizaciones internacionales. Programas de la Organización Internacional del Trabajo (OIT), de las Naciones Unidas, comisiones de la Unión Europea o estructuras intersectoriales como la Unidad de Exclusión Social del Gobierno británico de Tony Blair, estructuraron sus agendas sociales sobre tales conceptos (MacPherson, 1997; Porter, 2000; Davies, 2005). Anteriormente, sin embargo, había aparecido en la política social francesa (Lenoir, 1974) para indicar a ciertos grupos sociales en los que la política estatal debía poner atención. El extendido uso de la distinción inclusión/exclusión en el diseño de políticas radica en que permite introducir un criterio estratégico-político fácilmente entendible y transversalmente aplicable: las personas están dentro o fuera de criterios específicos de consideración social, por sobre o por debajo de ciertos límites que materializan la diferencia.

Si bien esta fórmula binaria puede ser útil en el diseño de políticas, en tanto aporta orientaciones concretas para decidir intervenciones y también para mostrar resultados, desde un punto de vista sociológico, una diferencia estricta entre inclusión y exclusión parece más una ficción con finalidades instrumentales que una observación de acontecimientos sociales reales. En su versión estática, la distinción transforma procesos (por ejemplo, participación versus marginalización) en estados (inclusión versus exclusión), adosa categorizaciones sociales a personas (excluidos versus incluidos), y

$\square$ Este artículo forma parte de las actividades de investigación de los proyectos Redes 130008, Fondo Nacional de Desarrollo Científico y Tecnológico (FONDECYT) 1140344, 1110437, y Núcleo Milenio Modelos de Crisis (NS130017), ICM-Iniciativa Científica Milenio. genera una demarcación de límites que impide concebir constelaciones paradójicas en las que se entremezclan diversas modalidades de inclusión y exclusión (Goodin, 1996; Davies, 2005; Sánchez, 2012).

El objetivo de este artículo es revisar la distinción inclusión/exclusión para intentar superar sus propias limitaciones, mostrar las paradojas que oculta e identificar conceptualmente diversas formas de inclusión/exclusión que puedan referir a situaciones concretas. La hipótesis que subyace a este ejercicio es que en sociedades modernas existen prácticas sociales de distinta naturaleza, que producen paradojas inevitables y que se vuelven invisibles cuando se emplea la distinción inclusión/ exclusión en su versión estática. A objeto de hacer frente a estas paradojas, es necesario identificar qué condiciones de inclusión hay en la exclusión y qué condiciones de exclusión existen en la inclusión. De este ejercicio deben emerger diversas constelaciones de inclusión/exclusión que permitan alejarse de una comprensión estática de la distinción, comprensión que más bien refuerza las condiciones presentes de inclusión y exclusión que se procura superar.

Para hacer plausible tal hipótesis, después de la Introducción, en este artículo se reconstruye la distinción inclusión/exclusión en la tradición sociológica (Simmel, Durkheim, Parsons), con énfasis en las paradojas que ella muestra y oculta (sección II). Se prosigue, en la sección III, con la elaboración sistémica de la distinción (Luhmann, Stichweh), para luego internarse en la propuesta de la escuela francesa (Fitoussi, Rosanvallon) y con ello mostrar los límites de la distinción concebida de manera estática ante la emergencia de nuevas desigualdades (sección IV). Sobre esta base, en el artículo se propone una clasificación original de distintas constelaciones de inclusión/exclusión y se ilustran con determinadas situaciones concretas, según puede apreciarse en la sección V. El documento concluye en la sexta sección con una síntesis de los principales hallazgos y sus consecuencias conceptuales y de políticas públicas. 


\section{II}

\section{Paradojas de la inclusión/exclusión en la tradición sociológica: Georg Simmel, Émile Durkheim, Talcott Parsons}

En la historia del pensamiento sociológico, la distinción inclusión/exclusión ha sido principalmente vinculada a la tradición funcionalista, desde Durkheim hasta Luhmann (Rawal, 2008; Braeckman, 2006; Allman, 2012). Sin embargo, fue Georg Simmel quien originalmente advirtió el carácter paradójico de la relación entre inclusión y exclusión. Sus ensayos sobre el extranjero y el secreto ilustran de manera distinta este punto. El extranjero no está adentro ni afuera, o puede estar adentro y afuera al mismo tiempo. Pertenece al grupo social, pero viene desde más allá; desarrolla encanto y significancia, pero no es "propietario de un espacio propio", ni en el sentido físico, ni en términos de referencia social (Simmel, 1950a, pág. 403). Esta ambigüedad mueve al grupo social a una apertura hacia él, desde la confianza hasta la confesión, pero a la vez el extranjero mantiene su libertad práctica. En otras palabras, el extranjero no está incluido ni excluido: es incluido como excluido.

El secreto funciona en un sentido más radical: incluye a todos los que saben; el resto está excluido. Este uso binario de la distinción oculta paradojas. El secreto implica un fuerte sentimiento (expectativa) de diferenciación entre personas basado en la posesión del conocimiento como propiedad interna del grupo social de pertenencia (Simmel, 1950b). La inclusión en el secreto contribuye al desarrollo de la identidad de grupo y a establecer sus límites. No obstante, existen grupos inclusivos (nación, credo) para los cuales el secreto consiste en "incluir a todos aquellos que no están explícitamente excluidos" (Simmel, 1950b, pág. 369). En este caso, Simmel realiza una aplicación binaria de la distinción: "Al principio de que está incluido aquel que no está explícitamente excluido, se le opone aquel otro de que está excluido quien no está explícitamente incluido" (Simmel, 1992, pág. 447). Esto hace que no se visualicen las paradojas reales. En cuestiones políticas, por ejemplo, la inclusión que lleva a cabo un Estado excluye inicialmente a miembros de otros Estados (hasta que se hayan "nacionalizado" o adquirido alguna ciudadanía formal): los excluye en la inclusión. Y en cuestiones religiosas, la inclusión a través de la tolerancia implica aceptación de otro al que de todos modos se le excluye como igual. Inclusión y exclusión aparecen más bien como formas interpenetradas.

En el caso de Durkheim, la conceptualización de la inclusión/exclusión puede ser reconstruida desde sus análisis sobre la solidaridad y la anomia. La sociedad moderna implica un orden de inclusiones y exclusiones a la vez: inclusión como solidaridad orgánica, exclusión como ámbitos distintos de división del trabajo (Durkheim, 2001). Ello produce problemas de integración nuevos, que se ven bien reflejados en las formas de suicidio (Durkheim, 2002). Paradójicamente, el suicidio egoísta y anómico (falta de integración) invita al suicida a seguir un patrón social de suicidio por el cual este expresa su negación de la sociedad (un lugar solitario, un medio, una escenificación, una nota). Paradójicamente, el suicida tiene que reconocerse a sí mismo como miembro de la sociedad para negar a la sociedad, de otro modo sería solo una "persona muerta", no un suicida. Por lo tanto, el suicida está incluido en su (último acto de) exclusión. Por otra parte, el suicidio altruista (sobreintegración) constituye un rechazo a la sociedad en nombre de la sociedad. Para designar esos actos de autosacrificio, la sociedad desarrolla las semánticas del mártir, el héroe, el santo (Luhmann, 1993), que afirman el acto como deseable: sustentan (y valoran positivamente) la exclusión como un acto de inclusión.

Parsons va un paso más allá que Simmel y Durkheim al dar a la distinción inclusión/exclusión un significado sociológico explícito: "El proceso mediante el cual los grupos previamente excluidos logran adquirir ciudadanía o membresía plena en la comunidad social, será llamado en este texto inclusión" (Parsons, 1965, pág. 1.015). De un modo más técnico, Parsons detalla esta definición: "En vista de la estructura plural de las sociedades modernas es altamente posible que personas u otras unidades, que en algunos aspectos son 'outsiders', sean incluidos junto a los 'locales' en otros aspectos [...] precisamente debido a la estructura plural de las sociedades modernas, la inclusión no es una cuestión de esto/lo-otro, sino una con múltiples componentes parciales de membresía y aceptación" (Parsons, 2007, págs. 73 y 74). 
Este autor atribuye a la inclusión el valor positivo, esperable, normal; la exclusión es el lado negativo. El punto de referencia empírico original para esta elaboración es lo que él denomina la situación de los "negros en América" (Parsons, 1965). En este contexto, el concepto de inclusión es empleado predominantemente como sinónimo de ciudadanía plena. Parsons (1965, pág. 1.026) entiende esto como una cuestión de "aceptación", esto es, "la capacidad y oportunidad de participación plena sin discriminación informal". La exclusión supone una derrota de este objetivo. Sin embargo, paradójicamente, la exclusión es rara vez exclusión plena: puesto que la ciudadanía plena es un ideal, la ciudadanía real incorpora exclusiones si se observa desde el ideal. Por ello, Parsons habla de múltiples componentes parciales de membresía y aceptación, porque finalmente la inclusión es un resultado acumulativo e incremental, lo que implica que en cada momento de ese proceso hay que contar con exclusiones por superar (Mascareño, 2012a).

La distinción inclusión/exclusión en su interpretación binaria queda por tanto superada por lo que ella misma invita a pensar. Nunca hay un estado único ni completo de inclusión o exclusión, sino situaciones en las que ambas condiciones se expresan a la vez.

\section{III}

\section{Inclusión y exclusión en la sociología sistémica: Niklas Luhmann y Rudolf Stichweh}

En el caso de la sociología sistémica contemporánea, se da un paso adelante en la identificación de situaciones complejas de inclusión/exclusión, aunque aún existe una tendencia a permanecer en el esquema binario de observación, lo que nuevamente induce a la sublimación de ciertas paradojas.

El análisis de la distinción inclusión/exclusión en Luhmann tiene un sentido preciso e innovador: "Inclusión (y análogamente exclusión) puede referirse solo al modo y la manera de indicar en el contexto comunicativo a los seres humanos, o sea, de tenerlos por relevantes" (Luhmann, 2005, pág. 229). En otro lugar, el autor aclara: "Lo que más bien quiere indicarse es que el sistema sociedad predispone a las personas asignándoles lugares en cuyo marco pueden actuar de acuerdo a expectativas complementarias" (Luhmann, 2007a, pág. 492). En tanto que para este autor lo social se define radicalmente por la vía de la comunicación, los individuos como tales están excluidos de lo social. Su única forma de participar de la sociedad aparece cuando en la comunicación son tomados en cuenta. Esto es lo que Luhmann quiere decir con "indicar" y "tenerlos por relevantes": la comunicación social los incorpora en tanto "personas". Esta incorporación puede adquirir diversas formas según el sistema de comunicación de que se trate - las personas votan, compran, venden, son sujetos de derecho, se enamoran-. En todos estos casos hay inclusión. De este modo, la inclusión no es una, no se trata de un esquema de observación de clase (arriba/ abajo) y no es solo de bienestar: también los subalternos están incluidos como posibilidad de subversión del orden y en esa medida son observados y controlados; a los deudores igualmente se les asigna un lugar relevante en la comunicación económica, más aún si dejan de pagar; y quienes quebrantan el derecho pueden ser excluidos de múltiples comunicaciones, pero al menos tienen que ser incluidos en el sistema penitenciario.

Lo que se gana con este concepto de inclusión/ exclusión referido a sistemas es la constatación de que tanto la inclusión como la exclusión de personas nunca son un fenómeno absoluto: nadie está plenamente incluido ni plenamente excluido. Es cierto que siempre se puede estar incluido como público potencial de un sistema del que no se participa formal o profesionalmente: los museos, los conciertos, el arte en general, esperan más público los fines de semana, pero esa participación es cualitativamente distinta a la del curador, del músico o del artista, y si a ambas cosas se les denomina "inclusión", entonces el concepto no es lo suficientemente preciso para captar la diferencia en las operaciones sociales reales. El público no puede "tocar": está incluido en la exclusión de la inclusión del arte, y eso es muy distinto a estar incluido o excluido a secas.

En vez de abrir la distinción a la designación de estas paradojas, Luhmann (2005 y 2007a) enfatiza la radicalidad de la distinción y habla del ámbito de la inclusión y del ámbito de la exclusión. El primero tiene una integración baja, en el sentido de que la inclusión 
en un ámbito no determina la inclusión en otros; el segundo, tiene una integración alta, en tanto que la exclusión de uno puede implicar exclusiones en cadena. La afirmación es lógica cuando se presupone que el principio de la diferenciación funcional predomina casi plenamente: tener dinero no tendría por qué significar tener poder político o más derechos (baja integración de la inclusión); y carecer de trabajo implica dificultades para la inclusión en educación, salud, vestimenta, entre otros (alta integración de la exclusión). El problema es que con ello se subvalora la potencialidad que aún poseen los principios sociales de estratificación y segmentación para alterar las inclusiones/exclusiones funcionales.

Mucho de lo que se denomina "poder fáctico" se explica por integraciones de la inclusión producto de la persistencia de la estratificación (el dinero puede pagar votos y decisiones jurídicas); y la ausencia de trabajo formal se puede reemplazar por trabajo informal (con lo que la exclusión se desacopla de impuestos y leyes laborales) o por participación en redes informales que permiten accesos indirectos a rendimientos funcionales a través de grupos segmentarios (Mascareño, 2014). Si a uno de estos ámbitos se le denomina inclusión y exclusión al otro, se establece una diferenciación binaria de estas situaciones que no se condice con las situaciones concretas. El esquematismo se lleva hasta un extremo cuando a la inclusión y a la exclusión se les atribuyen zonas geográficas: la ciudad "formal" donde impera el Estado de derecho y la favela (Luhmann, 2005, págs. 244 y 245).

Lo paradójico es que Luhmann puede observar estos problemas tanto en un nivel empírico como en uno teórico: "La diferencia inclusión/exclusión nunca se da empíricamente con tanta claridad como para que todas las personas puedan ser inequívocamente asignadas a uno de sus lados" (Luhmann, 2005, pág. 246). La pregunta es entonces por qué mantener la binariedad de la distinción como esquema de observación y no deconstruirla en los términos que lo exige la variedad de formas de inclusión/ exclusión presentes en la sociedad moderna ${ }^{1}$.

Rudolf Stichweh (2005, págs. 20-41) se orienta hacia este objetivo cuando identifica diversas formas de inclusión realizadas por sistemas funcionales: la inclusión por medio de relaciones profesional/cliente (salud, educación, derecho), de opciones de salida y voz (voicelexit-options) en política, economía, arte y

\footnotetext{
${ }^{1}$ Esto había sido anticipado correctamente por Robles (2005), aunque en su propuesta pone demasiado énfasis en la individuación como problema de referencia, con lo que pierde otras fuentes sociológicas que tensionan las relaciones de inclusión y exclusión.
}

medios), de inclusión bajo el papel de productor de rendimientos y público a la vez (relaciones íntimas), y de inclusión indirecta (por ejemplo, en la ciencia a través de la formación en el sistema educacional). Todo ello exige determinar las formas de inclusión de públicos. En la mayoría de los sistemas, los productores de rendimientos funcionales son a la vez públicos del sistema: los legisladores también votan y se someten al derecho, los vendedores también compran. Especialmente en el derecho se produce otra forma de inclusión de públicos: la cooptación de los acusados, es decir, de quienes están obligados (expresamente contra su voluntad) a participar de la comunicación jurídica. Y en otros ámbitos, los públicos se integran en roles secundarios (servicios voluntarios, ciencia y música amateur, deportes masivos), es decir, participan restringidamente (localmente y de manera no especializada) en la comunicación del sistema.

Como fácilmente se puede advertir, ninguna de estas formas de inclusión tiene aspiraciones de plenitud. Ya la inclusión como opción de salida (es decir, de restarse a la participación sistémica) en política, economía, arte y medios muestra que la inclusión puede paradójicamente realizarse como autoexclusión, esto es, cuando alguien - teniendo la oportunidad de hacerlo de otro modo- se niega a votar, a comprar o vender, a ser espectador del arte o audiencia de medios. Por otra parte, la inclusión indirecta implica la exclusión del propio sistema para incluirse en él a través de otro - como en el caso de la inclusión en rendimientos funcionales vía redes informales para aquellos que se encuentran en condiciones de subinclusión-. En el caso de la inclusión de públicos, ciertamente la inclusión por cooptación entraña exclusión de otras opciones. Y la inclusión en papeles secundarios supone a la vez exclusión de operaciones centrales del sistema. Se trata de formas de exclusión internas a los sistemas, en otras palabras, de inclusión en la exclusión. Así, las personas no están incluidas ni excluidas, tampoco en una posición intermedia, sino que, en la situación concreta en la que operan, se encuentran en ambas situaciones a la vez.

Más recientemente, Stichweh (Stichweh y Windolf, 2009, págs. 38-40) ha reformulado este problema, haciéndose cargo de situaciones paradójicas de inclusión/ exclusión a través de la distinción entre exclusión incluyente e inclusión excluyente. Con ello, la distinción se reintroduce en sí misma y deja en mejores condiciones de visibilizar paradojas: las instituciones de ayuda a jóvenes constituyen una exclusión incluyente en tanto limitan grados de libertad para apuntar luego a la "reinserción social", mientras que las bandas juveniles funcionan como inclusión excluyente en tanto integran al grupo 
por medio de una norma de oposición con estructuras de expectativas de la sociedad mayor. La misma diferencia podría aplicarse a grupos organizados de protesta (indígenas, ambientalistas, estudiantes) que generan potentes comunicaciones de exclusión social para hacer notar sus demandas de inclusión (exclusión incluyente), y a grupos terroristas que invitan a la participación en ellos, reforzando la irrevocabilidad e irreversibilidad de sus operaciones de exclusión (inclusión excluyente).

Con ello, la sociología sistémica da un paso decisivo hacia la "visibilización" de las paradojas en situaciones de inclusión/exclusión, y aporta una conceptualización más fina de ellas en casos de intervención. Pero aún la observación no es lo exhaustiva que debiera ser.

\section{IV}

\section{Inclusión y exclusión en la escuela francesa: Pierre Rosanvallon, Jean Paul Fitoussi}

La distinción inclusión/exclusión ha encontrado en Francia un uso extendido. La popularidad de los términos se expresa en la simbiosis existente entre su uso en el discurso político y en el teórico. En el primero de ellos, la figura clave es René Lenoir (1974), para quien los excluidos estaban constituidos por personas con todo tipo de discapacidades, enfermedades mentales, drogadictos, incluso ancianos y familias monoparentales. Entendida de este modo, la propuesta de Lenoir derivaba en los problemas característicos de la distinción inclusión/ exclusión aplicada de forma binaria: dualización de la sociedad en incluidos y excluidos, transformación de procesos en estados, atribución de categorizaciones (discriminatorias) a personas, ocultamiento de las paradojas en constelaciones reales. La distinción permitía ver la existencia de un problema, pero el esquematismo de su aplicación impedía una descripción adecuada.

Cuando Fitoussi y Rosanvallon (2010, pág. 27) afirman que la "dinámica social [...] no puede reducirse a quienes están 'adentro' y quienes están 'afuera'”, y cuando agregan a ello que la "exclusión es el resultado de un proceso, no un estado social dado", están precisamente distanciándose de una aplicación binaria de la distinción, con lo que se hace necesario determinar a qué situaciones sociales refiere ahora la distinción inclusión/ exclusión. La clave para entender este proceso no está en una desaparición de modalidades de estratificación y jerarquización clásicas (como enfatizaba Luhmann), sino en una fuerte individualización de sus formas, lo que impide la reconstrucción de una experiencia colectiva de cambio social. Si antes el estatus permitía reconocer la inclusión en un grupo, una clase, una comunidad, hoy esas referencias se vuelven inestables, por lo que a la descripción de identidades colectivas hay que sumar "trayectorias individuales y sus variaciones en el tiempo" (Fitoussi y Rosanvallon, 2010, pág. 31).

Para Fitoussi y Rosanvallon esto repercute en la forma en que los individuos experimentan sus relaciones con otros: i) quien antes era "un igual" (misma profesión, mismo espacio laboral, mismos lugares de residencia), ahora es distinto: "Un ejecutivo desempleado desde hace tiempo, por ejemplo, no vive su situación únicamente en términos de baja de los ingresos: se siente en primer lugar excluido del mundo de los ejecutivos" (Fitoussi y Rosanvallon, 2010, pág. 74), y ii) quien antes era distinto, ahora es un "igual desigual": "Entre un pequeño empresario en quiebra, un ejecutivo desocupado y un asalariado con un empleo precario, con el paso del tiempo las diferencias de ingreso terminan por borrarse, sin que pese a eso aquellos formen una categoría homogénea" (Fitoussi y Rosanvallon, 2010, pág. 76). Los autores llaman a esto nuevas desigualdades o desigualdades dinámicas (originadas en evoluciones técnicas, jurídicas y económicas) que se entrecruzan con la persistencia de desigualdades estructurales (de ingresos, patrimonio, educación).

La estrategia teórica de estos autores es observar la inclusión/exclusión bajo el esquema igualdad/desigualdad, con lo que en la observación de situaciones concretas pueden aparecer igualdades y desigualdades tanto en la inclusión como en la exclusión. Un primer rendimiento de esta estrategia es que las posibilidades descriptivas se multiplican y las paradojas reales se visibilizan: los que se hallan incluidos pueden estar desigualmente incluidos. Con ello se pueden observar distintos grados de exclusión en la inclusión, como sucede en un sistema educativo cuya calidad es altamente dependiente de las disponibilidades monetarias de las familias (Repetto, 
2011). O también: quienes están excluidos pueden ser objeto de inclusión igualitaria por la vía de desigualdades institucionalmente aceptadas que excluyen a otros, como en el caso de las cuotas de participación femenina en política (Ríos, 2008).

Un rendimiento adicional de esta estrategia es que la distinción igualdad/desigualdad introduce un estándar normativo en el análisis de inclusiones/exclusiones: se favorece a la igualdad como principio universal por sobre la desigualdad. Esto exige legitimación cuando la desigualdad es inherente a la situación: la desigualdad de profesiones (distintas profesiones), por ejemplo, es legítima, pero no lo es la de género (discriminación), y puesto que no lo es, entonces para igualar hay que introducir desigualdades justificadas (cuotas en política, flexibilidades en el espacio laboral, protección en la familia). Cuando esto no se hace, la inclusión en una categoría particular se vuelve excluyente, pues diferencia (jerarquiza) a personas que antes eran semejantes sin una justificación adecuada, trata a iguales como desiguales, hombres y mujeres frente a diferencias de salario a iguales trabajos, personas de diversa proveniencia étnica frente a los servicios públicos: "La acción de las desigualdades dinámicas genera diferencias en el entorno próximo, y en consecuencia, la no pertenencia social. Lo que está en juego en este proceso es una ruptura de la igualdad percibida como aún más intolerable porque parece no tener fundamento" (Fitoussi y Rosanvallon, 2010, pág. 103).

A esta perspectiva subyace el paradigma de la solidaridad presente en la sociología francesa desde Durkheim. En ese contexto, exclusión es un debilitamiento de los lazos que mantienen unida a la sociedad, e inclusión designa los esfuerzos intencionales (especialmente del Estado) por sostener esa unidad. Según Rosanvallon (2000), la combinación de esta perspectiva con las nuevas desigualdades se tematiza como nueva cuestión social: "Dos problemas centrales aparecen en el trasfondo de la crisis: el primero de ellos referido a la desintegración de los principios tradicionales de solidaridad; y el segundo a la inadecuación de los 'derechos sociales' como un marco en el cual resolver los problemas de exclusión social y de programas de asistencia que sean plenamente legítimos" (Rosanvallon, 2000, pág. 4).

El problema en este caso es un Estado de bienestar pasivo producto de la disociación de bienestar y trabajo. En la economía de mediados del siglo XX, los trabajadores podían tener una posición en la estructura laboral en pequeños nichos de productividad. Su posición desaventajada era compensada por el Estado. Cuando su situación laboral cambia con las condiciones de liberalización y competencia de las décadas finales del siglo XX, los trabajadores pierden sus nichos de empleo y solo quedan dependientes de la ayuda estatal: "Han llegado a ser desempleados pagados", y las políticas de bienestar se transforman en "un sistema de exclusión subvencionada" (Rosanvallon, 2000, págs. 61-63). Esto es lo que se podría denominar una inclusión compensatoria, que legitima formas distintas de exclusión.

Para advertir la novedad de esta situación, se requiere abandonar el antiguo paradigma de la exclusión como categoría estable y atender a los "quiebres, retrocesos y fallas que han experimentado [los individuos], a las desviaciones y diferencias que los marcan" (Rosanvallon, 2000, pág. 98). Se trata entonces de atender a procesos de exclusión antes que a estados. En esto adquiere alta relevancia entender la exclusión en un contexto de riesgos globales y tomar medidas frente a ello (Fitoussi y Stiglitz, 2009).

Como se aprecia, la escuela francesa también se esfuerza por visibilizar las paradojas en las situaciones concretas de inclusión/exclusión contemporáneas y aportar un marco de conceptualización dinámico para observarlas.

A continuación, se sistematiza ese marco por medio de la distinción conceptual de diversas constelaciones de inclusión/exclusión que debieran hacer visibles las paradojas y permitir un análisis más fino de ellas.

\section{V}

\section{Constelaciones de inclusión y exclusión} en la sociedad moderna

Cualesquiera sean las formas y combinaciones de inclusión y exclusión de que se trate, dos consideraciones parecen ser siempre relevantes a partir de las teorías analizadas: i) la primera es relativa a la individualización de los procesos de inclusión/exclusión y se interroga por la capacidad de selección de las personas en relación con las opciones que ellas consideren más apropiadas para la realización de sus planes de vida; ii) la segunda se vincula 
a los contextos sociales generales en que los procesos de inclusión/exclusión tienen lugar y su problema principal es la capacidad de distintos niveles de organización social de ofrecer tanto un número suficiente de alternativas, como una adecuada diversidad de ellas, de modo tal que las opciones de las personas sean cubiertas (en profundidad y amplitud) por la disponibilidad institucional. En el gráfico 1 se intenta ofrecer una representación de estas dos macrovariables y de las distintas constelaciones de inclusión/exclusión propuestas.

GRÁFICO 1

Dimensiones y situaciones de inclusión/exclusión

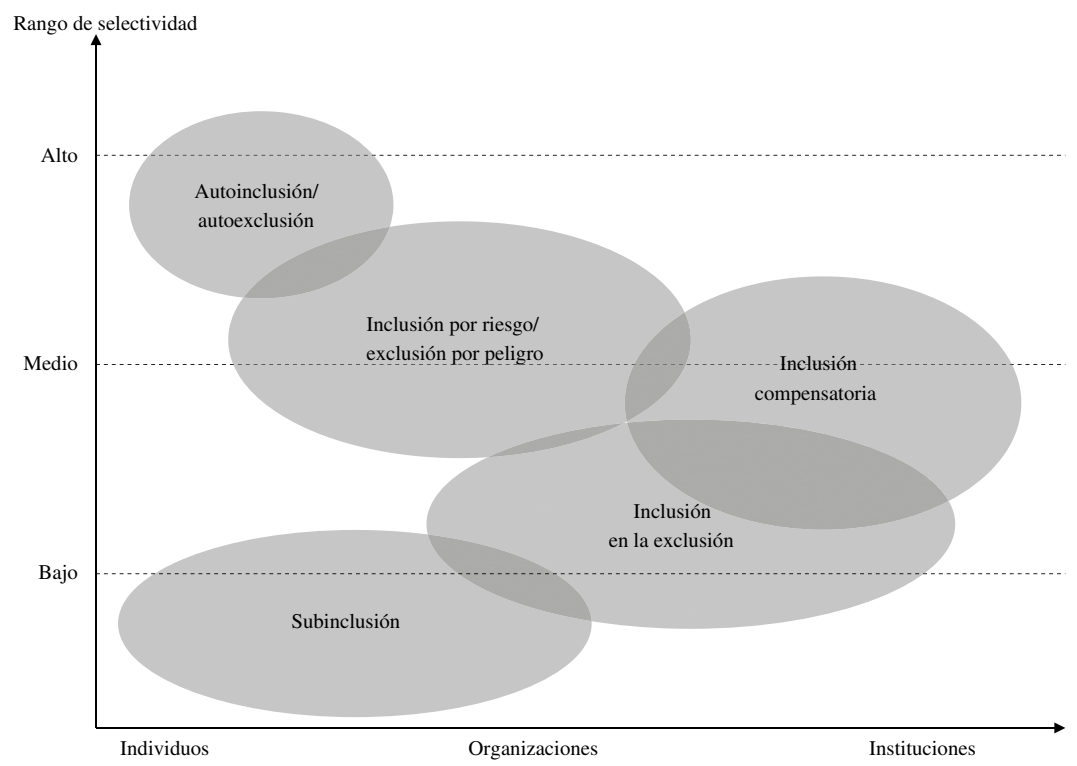

Fuente: elaboración propia.

- Autoinclusión/autoexclusión. Una situación escasamente considerada en las teorías de inclusión/ exclusión es aquella en la que los individuos eligen de manera autónoma participar o no participar en algún rendimiento social. El ejemplo más nítido de esto lo constituye la participación religiosa. Quien no se considera creyente $-36 \%$ en el mundo, incluidas personas no-religiosas y ateos (wIN-Gallup International, 2012) - se permite una autoexclusión de la práctica religiosa que, en todo caso, permanece disponible para futuras incorporaciones. Esto no obsta para que, observado desde la religión, este individuo: i) sea considerado incluido (como hermano, prójimo, hijo de Dios, o como infiel, es decir, una forma de inclusión por cooptación donde el incluido no entrega su consentimiento de inclusión), y para que, además, ii) las operaciones del sistema se orienten a su inclusión efectiva (evangelización, compasión o muerte de los infieles, aunque esta última forma revela una situación extrema de inclusión excluyente, como en el caso del Estado Islámico). Por todo ello, la posibilidad de revertir la autoexclusión en forma de autoinclusión siempre está disponible: se puede volver a creer o comenzar a hacerlo alguna vez. Para que situaciones de autoinclusión/autoexclusión sean posibles, las personas tienen que disponer de las capacidades individuales necesarias para identificar y proveerse de los medios destinados a sus fines (en términos clásicos: niveles educacionales y de ingreso medio por lo menos ), y convergentemente también distintos contextos sociales deben proporcionar un ambiente de diversidad y selección, de manera tal que si una opción no puede ser realizada, otra equivalente esté a disposición sin que ello implique cambios sustantivos en los planes de vida individual. Esta convergencia de condiciones individuales y sociales permite que si alguien deja un trabajo (autoexclusión) o, incluso más, si lo pierde, pueda acceder a un conjunto de otras posibilidades 
abiertas en forma de trabajos equivalentes elegibles (autoinclusión); o si el lugar de estudio por el que se optó (autoinclusión) no cumple con las expectativas trazadas, no solo el individuo pueda disponer de los medios (cognitivos, monetarios) para acceder a otro, sino que la sociedad también provea espacios de condiciones equivalentes.

En situaciones de falta de empleo como las descritas por Rosanvallon ("desempleados pagados", "exclusión subvencionada"), y ante la persistencia de desigualdades estructurales, esta posibilidad de autoexclusión/autoinclusión queda restringida a estratos superiores de la sociedad. Solo a este nivel se puede tener la expectativa de ser comunicativamente relevante para sistemas sociales y ver esa expectativa realizada por medio de ofertas de ahorro y crédito en bancos, de seguros privados en salud, de becas universitarias, o incluso de un nuevo y mejor empleo. Una alta capacidad de selección y decisión personal debe ser presupuesta, así como una oferta institucional capaz de entregar variedad de alternativas. La autoinclusión/autoexclusión relaciona a personas con los medios materiales y cognitivos para moverse en un ambiente institucional variado. De todos modos, esto no implica un estado de inclusión permanente. Los rangos de selectividad individual pueden reducirse rápida y drásticamente si un alto ejecutivo pierde su empleo y el contexto de crisis social no ofrece alternativas a ese nivel. Esto desencadena la alta integración de la exclusión de la que habla Luhmann (2005). En un contexto social monetarizado, por ejemplo, un desempleo relativamente permanente significa resolución de la hipoteca, traslado de hijos de colegio, cambio de seguros de salud y desestructuración de las referencias identitarias de los afectados, lo que reintroduce el problemas de las nuevas desigualdades sobre las que Fitoussi y Rosanvallon (2010) llaman la atención.

- Inclusión por riesgo y exclusión por peligro. Mientras que la autoinclusión/autoexclusión pone el acento en la movilidad de opciones individuales frente a la contingencia social, en el caso de la inclusión por riesgo/exclusión por peligro el énfasis se pone en las decisiones organizacionales. La compulsión de decisión deriva de un entorno de creciente complejidad en el que se hace necesario optar por alternativas. Pero los efectos no pretendidos de las decisiones organizacionales siempre se amplifican a límites difíciles de determinar: préstamos hipotecarios de alto riesgo a clientes locales (subprime) pueden desatar una crisis financiera global; innovaciones en sistemas tecnológicos de comunicación pueden emplearse para una invasión de la privacidad; decisiones de ahorro en instalaciones industriales pueden ocasionar catástrofes ecológicas; políticas fiscales deficientes pueden desencadenar desempleo de largo alcance, drásticas limitaciones en planes de vida o forzar migraciones masivas.

Como efecto de estos hechos, un alto número de personas quedan en una situación altamente ambigua: son excluidos de procesos organizacionales de toma de determinadas decisiones que eventualmente pueden afectarlos, pero son incluidos por esas decisiones como potenciales afectados por las consecuencias de ellas. Este problema se acrecienta con la transnacionalización de las organizaciones, en tanto los responsables de decisiones que afectan localmente pueden estar fuera de la jurisdicción de las autoridades del territorio en el que sus decisiones originan peligros ${ }^{2}$. Por otra parte, decisiones de carácter técnico (subir o bajar tasas de interés, implementar innovaciones tecnológicas, ahorrar en materias de seguridad) tienen extensas consecuencias normativas cuando se toman en cuenta alteraciones no buscadas en los planes de vida de las personas, o cuando se consideran los costos políticos asociados a ello. Las organizaciones pueden asumir el riesgo de tomar una decisión, pues se asume que desde un punto de vista estratégico, técnico e incluso político, es la mejor decisión que se podía tomar dada la información con que se contaba. Pueden incluso prepararse para posibles fallas mediante políticas de mitigación de consecuencias (inclusión compensatoria en forma de responsabilidad social empresarial). Pero aquel que está fuera del proceso de decisión y que no es consultado o de algún modo considerado en una decisión que irremediablemente le afectará, queda fuera de toda posibilidad de evitación o resguardo ante las consecuencias de la decisión. Esto ya no es riesgo, pues no hay opción de selección ante tales consecuencias; las personas quedan sujetas entonces al potencial peligro de una decisión ajena siempre riesgosa (Luhmann, 2006). En estos términos, puesto que riesgo es selección de una opción entre otras por medio de una decisión, las organizaciones e instituciones sociales formulan

2 Para esto solo se puede pensar en las disputas de inversión entre privados y Estados, o en la persecución de delitos informáticos (Mereminskaya, 2014; Shull, 2014). 
sus políticas desde el punto de vista de la inclusión en el riesgo de decidir. Pueden tener mecanismos de consulta a los potenciales afectados (como plebiscitos comunales o encuestas de usuarios) o procedimientos de inclusión de estos en el propio proceso de toma de decisiones (mesas de diálogo, presupuestos participativos, consejos comunales) (Mascareño, 2010). Sin embargo, con estos mecanismos nunca se logra alcanzar a todos los potenciales afectados (Karlsson, 2012). Siempre un grupo de ellos queda excluido de la decisión, o dicho de otro modo, queda incluido en el peligro de decisiones de otros. Esto constituye un serio problema democrático, pues la exclusión por peligro limita la concreción del derecho de las personas a dar su consentimiento en materias en que se verán potencialmente afectadas, y con ello aumentan las nuevas desigualdades (Fitoussi y Rosanvallon, 2010). Los afectados son incluidos en las consecuencias, pero excluidos de las decisiones, por ejemplo, ante el cambio unilateral de condiciones contractuales en materias comerciales (en la banca, el retail y las grandes casas comerciales) (Ossandón, 2012), en el cambio radical de un sistema de transporte público como aconteció con el Transantiago en Chile (Briones, 2009), o en el uso de territorios indígenas para explotación forestal o industrial sin consulta previa, como lo estipulan las regulaciones del Convenio sobre Pueblos Indígenas y Tribales, 1989 (Número 169) de la Organización Internacional del Trabajo (OIT) (Mereminskaya, 2011).

Los afectados participan bajo una forma de inclusión como cooptación. En el sentido de Stichweh (2005), son incluidos como público, pero sin la posibilidad de opciones de salida y voz (voicelexit-options) que les permitan deliberar en torno de las materias que les pueden afectar, ni de restarse a los efectos que pueden sobrevenir. Quedan en una especie de inclusión excluyente: atados a las consecuencias de un proceso que no promovieron ni aceptaron explícitamente.

- Inclusión compensatoria. La inclusión compensatoria es probablemente la forma más tradicional de inclusión y la más relacionada con un restablecimiento de condiciones de igualdad ante diferencias contingentes injustificables (por lo tanto, no legítimas). Definida en términos amplios, esta inclusión es aquella que instituciones sociales (generalmente públicas, pero no únicamente) llevan adelante (mediante políticas públicas, subsidios, legislación o acciones ad hoc) para equilibrar situaciones que se asumen temporales: enfermedades, desempleo de corto plazo, asistencia en vejez, pobreza o situaciones catastróficas (Fitoussi y Rosanvallon, 2010).

La inclusión compensatoria es, a la vez, una exclusión incluyente, en el sentido de que se identifica a un grupo desaventajado, desigual a otros, sobre el que se desarrolla una intervención orientada a la (re) inclusión. Dado que en la inclusión compensatoria (especialmente en la realizada por el Estado) se presupone que las situaciones de exclusión en las que se enfoca son transitorias, la permanencia de tales situaciones conduce a una exigencia de permanencia de la compensación. Este fue el problema que enfrentaron los Estados de bienestar en la segunda mitad del siglo XX. Las cargas monetarias de estos rendimientos estatales fueron lo que en la década de 1980 se definió como crisis del Estado de bienestar (Luhmann, 2007b; Offe, 2007), donde el desempleo jugó un papel central. El denominado desempleo estructural (Standing, 1983; Rodríguez, 1993) producía una exclusión estructural que requería una compensación estructural. Pero dado que los costos de la compensación se hacen cada vez mayores para el Estado, la calidad de la compensación no es satisfactoria para los individuos. La permanencia del desempleo produce una cadena de exclusiones en otros ámbitos (en habitación, educación, salud), que luego también se revierten en exigencias compensatorias hacia el Estado. La inclusión compensatoria se transforma así en una inclusión excluyente, en el sentido de que incluye a los afectados, pero lo hace en una situación de desigualdad, es decir, legitima e institucionaliza desigualdades. Por ejemplo, como acontece en el caso chileno, personas de ingresos medios y bajos tienen a disposición determinadas opciones educativas progresivamente restringidas en comparación con los niveles de ingresos más altos (Torche, 2005). Frente a esto, el Estado puede desarrollar políticas compensatorias como la entrega de subsidios o descuentos impositivos en educación (SII, 2013), los que sin embargo no alcanzan a equilibrar los rangos de selectividad de los afectados y provocan una estratificación en el acceso de distintas personas a rendimientos educativos de diversa calidad. Es decir, se produce una exclusión vinculada a la progresiva reducción del rango de selectividad individual (familiar), pero a la vez una inclusión estratificada en opciones educativas de calidad decreciente, $\mathrm{o}$ al menos variable. Cuando esto se hace rutinario y se le asocian semánticas de discriminación, hablamos 
de inclusión en la exclusión: se está incluido, pero de modo estratificado y estigmatizado, es decir, con exclusión de acceso estructural y semántico a niveles distintos ${ }^{3}$.

Por razones como esta, Rosanvallon (2000) ha preferido cambiar el enfoque de bienestar desde la idea de compensación fundada en derechos sociales a la idea de riesgo. Puesto que todos enfrentan riesgos distintos, la justificación política de la compensación se vuelve menos relevante: "[La noción de riesgo] Reemplaza la clásica idea de justicia, entendida como conformidad a la naturaleza, a una norma ética o política, con la idea de una justicia puramente contractual (el sistema de compensación)" (Rosanvallon, 2000, pág. 15). Es decir, la compensación reemplaza a la responsabilidad política por un acuerdo contractual. Lo que Rosanvallon no observa es que las operaciones de compensación del Estado dependen igualmente de decisiones que pueden hacerse con exclusión de los potenciales beneficiados/afectados $\mathrm{y}$, por tanto, sin atención a sus posibles daños futuros: la inclusión compensatoria los pone en peligro de exclusión. Los prolongados tiempos de espera para la atención de enfermedades potencialmente graves son un ejemplo de ello (Oliver y Mossialos, 2004); también el apoyo a sindicatos que promueven condiciones de acceso universal al trabajo y excluye a mujeres que requieren condiciones flexibles para una incorporación estable (Abramo, 2006).

En estos términos, la inclusión compensatoria funciona con paradojas controlables cuando las exclusiones que aborda son temporalmente limitadas; en cambio, cuando ellas son permanentes, las paradojas se multiplican y ya no se puede estar tan seguro de que las intervenciones estatales produzcan un bienestar generalizado. A este problema se abocan (y de él, en algunos casos, profitan) una serie de instituciones no gubernamentales, tanto nacionales como transnacionales, cuyo objetivo es poner límites a la cadena de exclusiones que puede tener lugar como consecuencia de una sistemática estratificación en el acceso producida por la inclusión compensatoria (organizaciones comunitarias, de iglesia, fundaciones privadas de asistencia, organizaciones internacionales de ayuda al desarrollo). Pero tal como el Estado, ellas también están sometidas a las paradojas en la inclusión compensatoria.

${ }^{3}$ Se vuelve sobre ello en "Inclusión en la exclusión".
Un problema mayor tiene lugar, sin embargo, cuando las compensaciones (públicas o privadas, nacionales o transnacionales) fallan o se vuelven rutinarias. En esos casos se consolidan situaciones de inclusión en la exclusión y subinclusión. Esto es lo que se muestra a continuación.

- Inclusión en la exclusión. Inclusión en la exclusión implica una condición de inclusión, pero en una posición de subordinación en comparación con otras categorías sociales. La inclusión en la exclusión supone una situación de asimetría entre grupos, justificada generalmente en términos tradicionales o comunitarios. La semántica y los discursos relativos a grupos juegan aquí un papel preponderante. Las teorías postcoloniales los denominan subalternos (Guha y Spivak, 1988): grupos discriminados por origen étnico, casta, clase, género, orientación sexual, lengua o religión. Sin necesidad de adoptar esta denominación, ni tampoco los componentes políticos vinculados a ella, lo cierto es que la inclusión en la exclusión se sustenta en discursos públicos que: i) forman categorías sociales de personas de acuerdo con ciertos rasgos que se entienden comunes, pero que no necesariamente forman parte de la autodescripción de estos grupos; ii) la estabilización pública de ese discurso conduce a una incorporación/adopción por parte del grupo de las categorías externamente formadas, y iii) la adopción de esas categorías sitúa a estos grupos en posición de subordinación frente al discurso dominante, que es reproducida por el mismo grupo en tanto asume su heterocategorización.

Probablemente, el ejemplo más claro de esto lo constituyen las categorías de género. Para Butler (2007, pág. 49), la propia categoría de mujer es insuficiente para captar "lo que una es [...] porque el género no siempre se constituye de forma coherente o consistente en contextos históricos distintos, y se entrecruza con modalidades raciales, de clase, étnicas, sexuales y regionales de identidades discursivamente constituidas". De ello se puede derivar que aun cuando la semántica de la mujer se emplee con objetivos emancipatorios (activismo), desde el interior de la denuncia fluye la subordinación: cada reivindicación de la mujer es una actualización de esa subordinación. La lucha por la inclusión excluye (Mascareño, 2013). Pero el problema no solo se expresa en términos de procesos de identificación. Tiene también consecuencias estructurales en la segregación laboral por género, en la concentración de mujeres en estratos bajos de la 
jerarquía ocupacional, y en las brechas salariales que resultan de estas clasificaciones (Mora, 2013; Uribe, 2008). Algo similar acontece con los migrantes. Especialmente cuando se trata de personas de baja calificación, sus condiciones de inclusión no solo vienen determinadas por formas de trabajo precario (esporádico, sin contrato, de bajos ingresos), sino que además cargan con discriminaciones étnicas, en particular cuando se trata de migración de países andinos (Mora, 2009). En estos casos hay inclusión, aunque con restricciones progresivas y sistemáticas en los rangos de selectividad que determinan distintos niveles de inclusión en la exclusión.

Un ejemplo sutil de inclusión en la exclusión, pero igualmente ilustrativo, es el de los procesos de identificación cultural ${ }^{4}$. En el marco de su crítica a un concepto esencialista de cultura que subyace a las posiciones del multiculturalismo liberal, Sergio Costa (2012) sostiene que el Estado moderno, al adherir a este enfoque, legitima la protección de minorías mediante resguardos y límites en cuyo interior las identidades puedan ser reproducidas sin el riesgo de una intervención externa que las diluya en asimilación. Con ello, el multiculturalismo liberal ya parte de supuestos paradójicos: que la identidad cultural no cambia (pues tiene un sustrato esencial inmutable), pero a la vez se asume que debe ser protegida para que no cambie (protección contra asimilación). De todos modos, sobre estos dos supuestos la Constitución del Brasil de 1988, formada en un ambiente político altamente progresivo, estableció dentro de sus garantías que sería reconocida la propiedad rural a quienes fuesen descendientes de antiguas comunidades de esclavos (quilombos). Hacia 1988 no existían grupos que demandaran este reconocimiento, pero el artículo constitucional estimuló movilizaciones políticas que vieron en esto una oportunidad para resolver problemas de tierra. En ellas, además, participaron una serie de agentes "externos": antropólogos, religiosos, activistas, políticos, agentes de Estado y medios de comunicación. En 2003, un decreto

${ }^{4}$ El fuertemente criticado enfoque de cultura de la pobreza (Lewis, 1975) podría también considerarse como inclusión en la exclusión. En su forma original, sin embargo, se la entiende como una producción endógena del grupo que se sostiene a sí misma transgeneracionalmente. Esto limita su comprensión como problema relacional en el que se atribuyen determinados rasgos, que luego otros grupos adoptan como autodescripción y en el que, además, se esconden las variables estructurales (especialmente las relaciones de subordinación) que determinan la producción de tales procesos de identificación (Harris, 2001). presidencial estableció que el procedimiento para declararse descendiente de esclavos sería la autoidentificación. Hacia fines de 2011, existían 3.524 comunidades descendientes de quilombos. Una de ellas era la comunidad de Mocambo, que siempre había manifestado una identidad rural no indígena: "Las ventajas legales [...] fueron persuasivas, y condujeron a los residentes de Mocambo, después de varias discusiones y disputas políticas, a aceptarse públicamente como descendientes de la comunidad quilombo" (Costa, 2012, pág. 150).

El ejemplo ilustra cómo el Estado procura originalmente la inclusión (acceso a tierras), pero para ello debe definir una identidad cultural que será beneficiada con la acción de inclusión. La relevancia simbólica de esa identidad (vinculada a la solución del problema de tierras) induce a una exclusión de otras posibilidades, con lo que el grupo queda subordinado a la forma de identificación construida por el Estado.

La inclusión en la exclusión hace evidente múltiples paradojas. Esta condición se incrementa cuando se entiende que ella se puede combinar con la exclusión por peligro (exclusión de grupos discriminados de procesos de decisión) o con la inclusión compensatoria (limitaciones de inclusión fundadas en discursos discriminatorios). O cuando se advierte que ella augura una entrada a situaciones de subinclusión como se revisa a continuación.

- Subinclusión. La subinclusión supone la ausencia de condiciones institucionales para hacer valer derechos fundamentales, pero la obligación de responder a un orden social que exige obediencia (Neves, 2006). Es decir, se niegan los derechos que están en la base de cualquier posibilidad de inclusión social positiva (participación en rendimientos institucionales u organizacionales), pero se reafirman las obligaciones que incluyen negativamente aquellas que amenazan con el uso de la fuerza física frente a la desviación de la norma. Estas obligaciones son particularmente reafirmadas para estos grupos, pues ante la imposibilidad de salir institucionalmente de las situaciones de subinclusión, los individuos pueden recurrir a la violencia, el delito, la ilegalidad o la subversión del orden general. No obstante, en muchas ocasiones la preocupación central de personas en situación de subinclusión es la mera subsistencia: refugiados, migrantes en zonas de guerra, perseguidos políticos, personas en situación de calle, extrema pobreza. En otras, determinados grupos son objeto de una 
convergencia acumulativa de discriminaciones que no solo limitan la accesibilidad universal a rendimientos institucionales, sino que además, dada su agrupación territorial, se los constituye en zonas en que el Estado de derecho carece de efectiva práctica y simbólica (poblaciones, favelas) (Mascareño, 2012b y 2014), o en grupos sistemáticamente impedidos del acceso a ciertos espacios o cargos en principio universales (indígenas, gays, migrantes). En tal sentido, la subinclusión se caracteriza por la negación de la autonomía individual y la restricción máxima de su rango de selectividad.

Para todos los que se mantienen en una situación de subinclusión, la aspiración de realización de los planes de vida por medio de instituciones compensatorias queda suspendida. En la historia de la sociología latinoamericana a esto se le denominó marginalidad, pobreza dura y pobreza extrema (Nun, 2001; Kessler y Di Virgilio, 2008), y se intentó superar mediante políticas compensatorias que, mientras la pobreza persiste, se deben considerar fracasadas. Ante el fracaso, pueden buscarse alternativas en espacios de informalidad para alcanzar algún nivel de (sub)inclusión, por ejemplo, en redes comunitarias, trabajos ocasionales, o mercado informal. Igualmente, la alternativa de participación en redes ilegales (de delincuencia, narcotráfico, corrupción) puede parecer atractiva, en tanto promete acceso a objetos y recursos que de otro modo no podrían ser alcanzados (Dewey, 2012). Lo que se obtiene con esto es un modo de inclusión alcanzado por vías generalmente ilegítimas, que en consecuencia no está sujeto a

\section{VI}

\section{Conclusión}

La diferencia entre inclusión y exclusión presenta un innegable valor para el análisis sociológico y de políticas públicas en sociedades complejas. Sin embargo, precisamente esa alta complejidad impide desarrollar este análisis en términos simples, atribuyendo un estado de inclusión a algunos y de exclusión a otros. El objetivo de este artículo ha sido elaborar una diferenciación analítica de distintas situaciones en que la inclusión y la exclusión se combinan, y hacerla plausible con ilustraciones y ejemplos de distinta naturaleza. Varias restricciones burocráticas, ni goza de algún tipo de protección social o jurídica. Se tiene acceso a algunos rendimientos necesarios para la subsistencia o para la realización de planes de vida por vías informales, pero al costo de entrar en un entorno desprovisto de protección por parte del derecho y de las instituciones formales, y por cierto, al precio de la persecución jurídica de todo acto punible. Sin duda, no solo las condiciones de subinclusión generan motivación suficiente para participar de redes informales ilegales. De hecho, en condiciones de subinclusión no se dispone de los recursos o vínculos suficientes para organizarlas. Estas siempre deben estar previamente formadas para que personas en situación de subinclusión sean cooptadas o decidan incluirse. Aun aquellos con los rangos de selectividad más amplios (autoinclusión/autoexclusión) pueden derivar hacia redes ilegales (para evasión de impuestos, colusión de mercado, sobornos en materias ambientales). También el espacio estatal responsable de inclusión compensatoria es objeto de este problema (corrupción, prebendas políticas, redes de favores, lobby no regulado). La diferencia está en que las personas en condiciones de subinclusión: i) tienen la ilegalidad como única alternativa; ii) son sindicadas como "responsables" cuando estas redes son descubiertas, y iii) permiten con ello que los niveles superiores de la red sigan operando y se rearmen luego de un tiempo. En tal sentido, la subinclusión es, además, funcional a los privilegios particularistas (informales, pero sobre todo ilegales) de otros. Permite su reproducción y a la vez su ocultamiento. conclusiones pueden ser extraídas de este ejercicio. Se diferencian a continuación en aquellas referidas al ámbito conceptual y las relacionadas con el diseño e implementación de políticas.

En términos conceptuales, las relaciones entre inclusión y exclusión vinculan expectativas individuales con rendimientos organizacionales o institucionales que determinan rangos de selectividad altos, medios o bajos para las personas. El rango más amplio de selectividad tiene lugar cuando las personas pueden decidir si participan o no 
(y cuándo hacerlo) de diferentes ofertas sociales. Se trata, por ejemplo, de personas de ingresos altos que pueden elegir una educación o salud privadas de mayor costo, u optar por el ahorro y acceder a alternativas públicas. Estos niveles de selectividad decrecen progresivamente para las otras situaciones de inclusión/exclusión. Sin procedimientos de consulta a los potenciales afectados (exclusión por peligro), las decisiones de organizaciones o instituciones alcanzan a personas que deben enfrentar las consecuencias con sus propios recursos o esperar formas de inclusión compensatoria (generalmente de organismos públicos), en las que el rango de selectividad disponible viene institucionalmente predefinido. La inclusión en la exclusión discrimina culturalmente y deja a las personas en situaciones de subordinación frente a contextos organizacionales o institucionales. La subinclusión, en tanto, evidencia el rango más restringido de selectividad para las personas. Esta implica, por una parte, la imposibilidad de concretizar los derechos fundamentales de cada individuo, y por otra, el sometimiento de ellos a las obligaciones que impone el orden jurídico.

Entre estas distintas formas de inclusión y exclusión puede haber una permanente movilidad, especialmente en las zonas fronterizas de la autoinclusión/autoexclusión y la inclusión compensatoria, o entre la inclusión en la exclusión y la subinclusión. Es decir, las personas se mueven en un espacio transicional que rara vez puede ser identificado con estados permanentes. Esto puede explicar lo que Araujo y Martuccelli (2011, pág. 168) denominan inconsistencia posicional en la estratificación social: "El sentimiento de que todo puede, todo el tiempo, cambiar". Incluso de una situación de subinclusión (la más extrema en restricciones a la selectividad) se puede salir por vías institucionales formales hacia una inclusión compensatoria y, en el marco de ella, sufrir las consecuencias de discursos discriminatorios de la inclusión en la exclusión. Por otra parte, la extendida capacidad de decidir de la autoinclusión/autoexclusión puede reducirse debido a contingencias del entorno (crisis financieras, enfermedades, accidentes), por decisiones propias (cambio en el sistema público de educación o salud por ahorro), o por decisiones de otros en las que se resulta afectado (expropiaciones, crisis de la empresa en la que se trabaja), lo que puede mover a estas personas hacia zonas de inclusión compensatoria para la concreción de determinadas expectativas. La complejidad deconstruye permanentemente la estratificación, pero no la elimina. De ahí que se puedan advertir diferencias de selectividad e inclusión, pero no se puedan fijar posicionalmente.
Dicho en otros términos, la distinción inclusión/exclusión no puede ser aplicada binariamente bajo condiciones de alta complejidad social.

Para el diseño e implementación de políticas públicas, esta debe ser una consideración central: no se puede seguir hablando de inclusión y exclusión como si se tratara de dos mundos separados, como si solo los subincluidos estuvieran excluidos, o que solo aquellos con capacidad de autoinclusión y autoexclusión estuvieran incluidos. Tampoco se puede identificar exclusión con extrema pobreza e inclusión con superación de la línea de pobreza: en el primer caso, se trata de subinclusión; en el segundo, de al menos inclusión compensatoria. La fórmula binaria inclusión/exclusión es demasiado rígida para este tipo de problemas. Tanto la movilidad en las zonas de frontera, como las múltiples dimensiones sociales en las que paralelamente se puede participar o no participar, hacen de esta fórmula una simplificación excesiva que trivializa el objeto de las políticas, distorsiona sus efectos e impide un diagnóstico adecuado de los problemas que se tratan.

Finalmente, cualquier intervención, sea pública o privada, debe ser consciente de sus potenciales resultados excluyentes. Esto es de especial relevancia cuando se diseñan e implementan programas solo sobre la base de modelos técnicos en que los futuros afectados no son considerados, o cuando los propios programas refuerzan condiciones estratificatorias de inclusión en la exclusión. No hay decisión sin consecuencias, y sobre todo con respecto a organizaciones e instituciones públicas el cálculo de posibles afectados por las decisiones nunca puede ser determinado con exactitud.

Es un hecho que la sinergia y complementariedad entre diversos programas sociales y políticas públicas en general son fundamentales para producir barreras a las diversas posibilidades de exclusión, pero también es cierto que cualquiera sea el marco de políticas siempre hay que contar con problemas de exclusión ante los cuales se debe reaccionar con flexibilidad. La primera condición con tal propósito es tener conceptos suficientemente finos para aplicar al diagnóstico de casos y a la evaluación de las consecuencias de cada decisión, tanto privada como pública. Una distinción gruesa entre inclusión y exclusión solo confunde y simplifica en exceso problemas que son complejos. Con el modelo aquí presentado, sobre la base de cinco situaciones interrelacionadas de inclusión/exclusión, se intenta aumentar la capacidad sociológica y política de observar y procesar esa complejidad. 


\section{Bibliografía}

Abramo, L. (ed.) (2006), Trabajo decente y equidad de género en América Latina, Santiago de Chile, Oficina Internacional del Trabajo (OIT).

Allman, D. (2012), "The Sociology of Social Inclusion", sAGE Open [en línea] http://sgo.sagepub.com/content/3/1/2158244012471957.

Araujo, K. y D. Martuccelli (2011), "La inconsistencia posicional: Un nuevo concepto sobre la estratificación social", Revista de la CEPAL, $\mathrm{N}^{\circ} 103$ (LC/G.2487-P), Santiago de Chile.

Braeckman, A. (2006), "Niklas Luhmann's systems theoretical redescription of the inclusion/exclusion debate", Philosophy \& Social Criticism, vol. 32, $\mathrm{N}^{\circ} 1$, SAGE.

Briones, I. (2009), "Transantiago: Un problema de información", Estudios Públicos, $\mathrm{N}^{\circ} 116$, Santiago de Chile, Centro de Estudios Públicos.

Butler, J. (2007), El género en disputa, Barcelona, Paidós.

Costa, S. (2012), "Freezing differences. Politics, law, and the invention of cultural diversity in Latin America", Legitimization in World Society, A. Mascareño y K. Araujo (eds.), Surrey, Ashgate.

Davies, J. (2005), "The social exclusion debate: Strategies, controversies and dilemmas", Policy Studies, vol. 26, $\mathrm{N}^{\mathrm{o}} 1$, Londres, Routledge.

Dewey, M. (2012), "Illegal police protection and the market for stolen vehicles in Buenos Aires", Journal of Latin American Studies, vol. 44, $\mathrm{N}^{\circ} 4$, Cambridge University Press.

Durkheim, E. (2002), Suicide, Londres, Routledge.

(2001), La división del trabajo social, Madrid, Akal.

Fitoussi, J.P. y P. Rosanvallon (2010), La nueva era de las desigualdades, Buenos Aires, Manantial.

Fitoussi, J.P. y J. Stiglitz (2009), "The ways out of the crisis and the building of a more cohesive world", Document de Travail, $\mathrm{N}^{\circ}$ 17, Centre de Recherche en Économie de Sciences Po.

Goodin, R. (1996), "Inclusion and exclusion", European Journal of Sociology, vol. 37, $\mathrm{N}^{\circ}$ 2, Cambridge, Cambridge University Press.

Guha, R. y G. Spivak (eds.) (1988), Selected Subaltern Studies, Oxford, Oxford University Press.

Harris, M. (2001), Antropología cultural, Madrid, Alianza.

Karlsson, J. (2012), "The boundaries of transnational democracy: Alternatives to the all-affected principle", Review of International Studies, vol. 38, $\mathrm{N}^{\circ}$ 2, Cambridge, Cambridge University Press.

Kessler, G, y M.M. Di Virgilio (2008), "La nueva pobreza urbana: Dinámica global, regional y argentina en las últimas dos décadas", Revista de la CEPAL, № 95 (LC/G.2382-P), Santiago de Chile.

Lenoir, R. (1974), Les exclus: un français sur dix, París, Seuil.

Lewis, O. (1975), Five Families: Mexican Case Studies in the Culture of Poverty, Londres, Basic Books.

Luhmann, N. (2007a), La sociedad de la sociedad, México, D.F., Herder.

(2007b), Teoría política en el Estado de bienestar, Madrid, Alianza Editorial.

(2006), Sociología del riesgo, México, D.F., Universidad Iberoamericana.

(2005), "Inklusion und Exklusion", Soziologische Aufklärung, $\mathrm{N}^{\circ}$ 6, Wiesbaden, vs Verlag.

(1993), Gesellschaftsstruktur und Semantik, Band 3, Frankfurt, Suhrkamp.

MacPherson, S. (1997), "Social exclusion", Journal of Social Policy, vol. 26, N $\mathrm{N}^{\mathrm{T}}$ 4, Cambridge, Cambridge University Press.

Mascareño, A. (2014), "Grenzen der Kontrolle: Institutionalisierung und Informalisierung des Raums. Das Beispiel Lateinamerika", Konstruktion und Kontrolle. Zur Raumordnung sozialer Systeme, P. Goeke, R. Lippuner y J. Wirths (eds.), Wiesbaden, Springer vs.
(2013), "Los varios rostros del género y sus fundamentos estructurales", Desigualdad en Chile: La continua relevancia del género, C. Mora (ed.), Santiago de Chile, Ediciones Universidad Alberto Hurtado.

(2012a), "The many faces of justice and its structural foundations", Legitimization in World Society, A. Mascareño y K. Araujo (eds.), Surrey, Ashgate.

(2012b), Die Moderne Lateinamerikas. Weltgesellschaft, Region und funktionale Differenzierung, Bielefeld.

(2010), "Coordinación social mediante políticas públicas. El caso chileno", Revista de la CEPAL, N 101 (LC/G.2455-P), Santiago de Chile.

Mereminskaya, E. (2014), Arbitraje comercial internacional en Chile Desafíos y desarrollo, Santiago de Chile, Thomson Reuters.

(2011), "El Convenio 169 de la OIT sobre pueblos indígenas y tribales. Derecho internacional y experiencias comparadas", Estudios Públicos, $\mathrm{N}^{\circ}$ 121, Santiago de Chile, Centro de Estudios Públicos.

Mora, C. (ed.) (2013), Desigualdad en Chile: La continua relevancia del género, Santiago de Chile, Ediciones Universidad Alberto Hurtado.

(2009), "Estratificación social y migración intrarregional: Algunas caracterizaciones de la experiencia migratoria en Latinoamérica", Universum, vol. 24, № 1 , Talca, Universidad de Talca.

Neves, M. (2006), "Die Staaten im Zentrum und die Staaten an der Peripherie", Soziale Systeme, vol. 12, № 2, Stuttgart, Lucius \& Lucius Verlag.

Nun, J. (2001), Marginalidad y exclusión social, Buenos Aires, Fondo de Cultura Económica.

Offe, C. (2007), Contradicciones en el Estado de bienestar, Madrid, Alianza.

Oliver, A. y E. Mossialos (2004), "Equity of access to health care: Outlining the foundations for action", Journal of Epidemiology and Community Health, vol. 58, $\mathrm{N}^{\mathrm{o}} 8$.

Ossandón, J. (ed.) (2012), Destapando la caja negra. Sociologías de los créditos de consumo en Chile, Santiago de Chile, Instituto de Investigación en Ciencias Sociales (ICSO).

Parsons, T. (2007), American Society. A Theory of the Societal Community, Boulder, Paradigm Publishers.

(1965), "Full citizenship for the negro American? A sociological problem", Daedalus, vol. 94, No 4, Cambridge, Massachusetts, The MIT Press.

Porter, F. (2000), "Social exclusion: What's in a name?", Development in Practice, vol. 10, No 1 , Taylor \& Francis.

Rawal, N. (2008), "Social inclusion and exclusion: A review", Dhaulagiri Journal of Sociology and Anthropology, vol. 2.

Repetto, A. (2011), "The effectiveness of private voucher education: Evidence from structural switches", Educational Evaluation and Policy Analysis, vol. 33, $\mathrm{N}^{\circ} 2$, junio.

Ríos, M. (ed.) (2008), Mujer y política. El impacto de las cuotas de género en América Latina, Santiago de Chile, Catalonia.

Robles, F. (2005), "Contramodernidad y desigualdad social: Individualización e individuación, inclusión/exclusión y construcción de identidad. La necesidad de una sociología de la exclusión", Revista Mad, No 12

Rodríguez, O. (1993), La teoría del subdesarrollo de la CEPAL, Buenos Aires, Siglo XXI.

Rosanvallon, P. (2000), The New Social Question. Rethinking the Welfare State, Princeton, Princeton University Press.

Sánchez, K. (2012), "Social exclusion, social cohesion: Defining narratives for development in Latin America", Journal of International Development, vol. $24, \mathrm{~N}^{\circ} 6$, Hoboken, John Wiley \& Sons.

Shull, A. (2014), "Global Cybercrime: The Interplay of Politics and Law", Internet Governance Papers $\mathrm{N}^{\mathrm{0}} 8$, Centre for International Governance Innovation. 
SII (Servicio de Impuestos Internos) (2013), "Rebaja de impuestos por gastos en educación" [en línea] http://www.sii.cl/pagina/ intermedia/beneficio_educacion/beneficio_educacion.html.

Silver, H. (1994), "Exclusión social y solidaridad social: Tres paradigmas", Revista Internacional del Trabajo, vol. 113, No 5/6, Ginebra, Organización Internacional del Trabajo (OIT).

Simmel, G. (1992), Soziologie, Frankfurt, Suhrkamp.

(1950a), "The stranger", The Sociology of George Simmel, K. Wolff (ed.), Glencoe, Illinois, The Free Press.

(1950b), "The secret and the secret society", The Sociology of George Simmel, K. Wolff (ed.), Glencoe, Illinois, The Free Press.

Standing, G. (1983), "El concepto de desempleo estructural", Revista Internacional del Trabajo, vol. 102, $\mathrm{N}^{\circ} 2$, Ginebra, Organización Internacional del Trabajo (OIT).
Stichweh, R. (2005), Inklusion und Exklusion. Studien zur Gesellschaftstheorie, Bielefeld, Verlag.

Stichweh, R. y P. Windolf (eds.) (2009), Inklusion und Exklusion: Analysen zur Sozialstruktur und sozialen Ungleichheit, Wiesbaden, vs Verlag.

Torche, F. (2005), "Privatization reform and inequality of educational opportunity: The case of Chile", Sociology of Education, $\mathrm{N}^{\circ} 78$.

Uribe, V. (2008), "Inequidades de género en el mercado laboral: El rol de la división sexual del trabajo", Cuaderno de Investigación, $\mathrm{N}^{\mathrm{o}}$ 35, Santiago de Chile, Dirección del Trabajo.

wIN-Gallup International (2012), "Global Index of Religiosity and Atheism" [en línea] http://www.wingia.com/web/files/news/14/ file/14.pdf. 